\title{
Some New Results for Struve Matrix Functions
}

\author{
Ayman Shehata
}

\begin{abstract}
This study deals with the convergence properties of Struve matrix functions within complex analysis. Certain new classes of matrix differential recurrence relations, matrix differential equations, the various families of integral representations and integrals obtained here are believed to be new in the theory of Struve matrix functions, and the several properties of the modified Struve matrix functions are also included. Finally, we investigate the operational rules which yield a different view of the expansion formulae for Struve and modified Struve matrix functions.
\end{abstract}

Mathematics Subject Classification (2010). Primary 33C10, 33C45; Secondary $15 \mathrm{~A} 60$.

Keywords. Struve and modified Struve matrix functions, Struve's matrix differential equation.

\section{Introduction and preliminaries}

Struve functions are mainly investigated because of their intrinsic mathematical importance in various problems in many parts of mathematical physics, physics and applied mathematics, and because of the fact that these functions are shown to be natural solutions of a particular set of ordinary and partial differential equations in various directions; they have a wide variety of applications in different branches of science and technology. The importance of studying special matrix functions and polynomials is with their applications in certain areas of applied mathematics, mathematical physics, physics, number theory, statistics, and engineering. In these fields, many properties, extensions, and generalizations of them have been published during the last years by many authors $[1,2,7,8,9,10,11,12,13,14]$. In particular, Struve matrix functions are also potentially useful related to the more familiar Bessel matrix functions. In fact, Mahmoud [15] has earlier introduced the Struve matrix function. Subsequently, motivated by the above-mentioned works, the importance of some interesting unified matrices associated with a variety of versions of Struve matrix 
functions in various problems in many places of mathematical physics, physics, applied mathematics, and engineering, have been clearly demonstrated in the language of matrices.

Motivated mainly by investigation of the Struve matrix function which is a matrix extension of the Struve scalar function, this study is organized as follows. The convergence, order, and type of Struve matrix function are investigated. Differential recurrence relations and Struve's matrix differential equation of $H_{A}(z)$ are given. Integral representations and some integrals of $H_{A}(z)$ are obtained in Section 2 . Numerous properties of the modified Struve matrix function are studied in Section 3. Various families of the expansion formulae with Struve matrix functions of the results presented by operational techniques as well as new and known generalizations are indicated in Section 4.

Throughout this study, for a matrix $A \in \mathbb{C}^{N \times N}, \sigma(A)$ denotes the set of all eigenvalues of $A$ and is called its spectrum. Furthermore, the identity matrix and the null matrix in $\mathbb{C}^{N \times N}$ will be denoted by $I$ and $\mathbf{0}$, respectively. We say that the matrix $A$ in $\mathbb{C}^{N \times N}$ is a positive stable matrix if $\operatorname{Re}(z)>0$ for all $z \in \sigma(A)$.

Definition 1.1. Jódar and Cortés defined the Gamma matrix function as follows (see $[5])$

$$
\Gamma(A)=\int_{0}^{\infty} e^{-t} t^{A-I} d t ; \quad t^{A-I}=\exp ((A-I) \ln t)
$$

where $A$ is a positive stable matrix in $\mathbb{C}^{N \times N}$.

If $\sigma(A)$ does not contain a zero or a negative integer, the matrix form of the Pochhammer symbol is defined by

$$
(A)_{n}= \begin{cases}A(A+I) \ldots(A+(n-1) I)=\Gamma(A+n I) \Gamma^{-1}(A), & n \in \mathbb{N} \\ I, & n=0\end{cases}
$$

where $\Gamma(A)$ is an invertible matrix in $\mathbb{C}^{N \times N}$ and $\Gamma^{-1}(A)$ is its inverse Gamma matrix function.

Definition 1.2. Jódar and Cortés defined the hypergeometric matrix function in the form (see [6])

$$
{ }_{2} F_{1}(A, B ; C ; z)=\sum_{k=0}^{\infty} \frac{z^{k}}{k !}(A){ }_{k}(B)_{k}\left[(C)_{k}\right]^{-1},
$$

where $A, B$, and $C$ are matrices of $\mathbb{C}^{N \times N}$ satisfying the condition that $C+n I$ is an invertible matrix for all integers $n \geq 0$ and for $|z|<1$.

Theorem 1.1. Let $A, B$ and $C$ be matrices in $\mathbb{C}^{N \times N}$ where the matrix $C$ satisfies the condition that $C+n I$ is an invertible matrix for all integers $n \geq 0$, and $C$, $C-A, C-B$ and $C-A-B$ are positive stable matrices where all matrices are commutative. Then the relation

$$
{ }_{2} F_{1}(A, B ; C ; 1)=\Gamma(C-A-B) \Gamma(C) \Gamma^{-1}(C-A) \Gamma^{-1}(C-B) .
$$

holds. 
Definition 1.3. Let $A$ be a matrix in $\mathbb{C}^{N \times N}$ satisfying (see [7])

$$
\sigma(A) \cap \mathbf{Z}^{-}=\emptyset \text {. }
$$

Sastre and Jódar defined the Bessel matrix function $J_{A}(z)$ as follows:

$$
J_{A}(z)=\sum_{k=0}^{\infty} \frac{(-1)^{k}}{k !} \Gamma^{-1}(A+(k+1) I)\left(\frac{1}{2} z\right)^{A+2 k I}, \quad 0<z<\infty .
$$

Theorem 1.2. [5] If $A$ is a matrix in $\mathbb{C}^{N \times N}$ satisfying $\operatorname{Re}(z)>0$ for all eigenvalues $z \in \sigma(A)$ and for $n \geq 1$ an integer, then

$$
\Gamma(A)=\lim _{n \rightarrow \infty}(n-1) !\left[(A)_{n}\right]^{-1} n^{A} .
$$

Fact 1.1. For an arbitrary matrix $A \in \mathbb{C}^{N \times N}$, the relation is (see [3])

$$
D^{k}\left[t^{A+m I}\right]=(A+I)_{m}(A+I)_{m-k} t^{A+(m-k) I}, \quad k=0,1,2, \ldots
$$

For $A \in \mathbb{C}^{N \times N}$, Legendre's duplication formula for the Gamma matrix function is as follows:

$$
\Gamma(2 A)=\frac{1}{\sqrt{\pi}} 2^{2 A-I} \Gamma(A) \Gamma\left(A+\frac{1}{2} I\right) .
$$

If $\operatorname{Re}(\mu) \in \sigma(A)$ is not an integer and using (1.2), we have the relation

$$
\Gamma(I-A-n I) \Gamma^{-1}(I-A)=(-1)^{n}\left[(A)_{n}\right]^{-1},
$$

where $\Gamma(I-A)$ is an invertible matrix.

\section{Definition and some new properties for Struve's matrix functions}

Definition 2.1. Let us suppose that $A$ is a matrix in $\mathbb{C}^{N \times N}$ satisfying the condition

$$
\operatorname{Re}(\mu)>-\frac{3}{2} \text { for every eigenvalue } \mu \in \sigma(A) \text {. }
$$

Then, we define Struve's matrix function by the series as follows

$$
\mathbf{H}_{A}(z)=\sum_{k=0}^{\infty} \frac{(-1)^{k}}{\Gamma\left(k+\frac{3}{2}\right)} \Gamma^{-1}\left(A+\left(k+\frac{3}{2}\right) I\right)\left(\frac{z}{2}\right)^{A+(2 k+1) I} .
$$

Theorem 2.1. For a matrix $A$ in $\mathbb{C}^{N \times N}$ satisfying (2.1), the Struve matrix function is an entire function and has order $\rho=\frac{1}{2}$ and type $\tau=0$.

Proof. The convergence properties of Struve's matrix function are given by the ratio test, and using (1.7) and (2.2), we have

$$
\begin{aligned}
& \lim _{k \rightarrow \infty}\left|\left\|\frac{(-1)^{k+1} \Gamma\left(k+\frac{3}{2}\right) \Gamma^{-1}\left(A+\left(k+\frac{5}{2}\right) I\right)\left(\frac{z}{2}\right)^{A+(2 k+3) I}}{(-1)^{k} \Gamma\left(k+\frac{5}{2}\right) \Gamma^{-1}\left(A+\left(k+\frac{3}{2}\right) I\right)\left(\frac{z}{2}\right)^{A+(2 k+1) I}}\right\|\right| \\
& =\lim _{k \rightarrow \infty} \frac{\left\|\left(A+\left(k+\frac{3}{2}\right) I\right)^{-1}\right\|}{\left(k+\frac{3}{2}\right)}\left|\left(\frac{z}{2}\right)^{2}\right| \leq \lim _{k \rightarrow \infty} \frac{\left\|(2 A+(2 k+3) I)^{-1}\right\|}{2 k+3}\left|z^{2}\right|=0,
\end{aligned}
$$


taking into consideration the well-known limits

$$
\lim _{k \rightarrow \infty}\left\|\frac{\ln \Gamma(A+k I)}{k \ln (k)}\right\|=1
$$

and

$$
\lim _{k \rightarrow \infty}\left\|\frac{[\Gamma(A+k I)]^{\frac{1}{k}}}{k}\right\|=\frac{1}{e} .
$$

Now, the order and type of the Struve matrix function are calculated in the following:

$$
\begin{aligned}
\rho & =\limsup _{k \rightarrow \infty} \frac{k \ln (k)}{\ln \left(\frac{1}{\left\|U_{k}\right\|}\right)}=\limsup _{n \rightarrow \infty} \frac{k \ln (k)}{\ln \left\|(-1)^{k} \Gamma\left(k+\frac{3}{2}\right) \Gamma\left(A+\left(k+\frac{3}{2}\right) I\right) 2^{A+(2 k+1) I}\right\|} \\
& \leq \limsup _{k \rightarrow \infty} \frac{1}{\left\|\frac{\ln \Gamma\left(k+\frac{3}{2}\right)}{k \ln (k)}+\frac{\ln \Gamma\left(A+\left(k+\frac{3}{2}\right) I\right)}{k \ln (k)}+\frac{(A+(2 k+1) I) \ln (2)}{k \ln (k)}\right\|}=\frac{1}{2},
\end{aligned}
$$

and

$$
\begin{aligned}
\tau & =\frac{1}{e \rho} \limsup _{k \rightarrow \infty} k\left(\left\|U_{k}\right\|\right)^{\frac{\rho}{k}} \\
& =\frac{2}{e} \limsup _{k \rightarrow \infty} k\left(\left\|\frac{(-1)^{k}}{\Gamma\left(k+\frac{3}{2}\right)} \Gamma^{-1}\left(A+\left(k+\frac{3}{2}\right) I\right) 2^{-(A+(2 k+1) I)}\right\|\right)^{\frac{1}{2 k}} \\
& =\frac{1}{e} \limsup _{k \rightarrow \infty} k\left(\frac{1}{\Gamma\left(k+\frac{3}{2}\right)\left\|\Gamma\left(A+\left(k+\frac{3}{2}\right) I\right)\right\|}\right)^{\frac{1}{2 k}} \\
& =\frac{1}{e} \limsup _{k \rightarrow \infty}\left(\frac{1}{\Gamma\left(k+\frac{3}{2}\right)}\right)^{\frac{1}{2 k}} \limsup _{k \rightarrow \infty} \frac{k}{\left(\left\|\Gamma\left(A+\left(k+\frac{3}{2}\right) I\right)\right\|\right)^{\frac{1}{2 k}}}=0 .
\end{aligned}
$$

The matrix differential recurrence relations presented in the following theorem are also interesting:

Theorem 2.2. For matrices $A$ and $A-I$ in $\mathbb{C}^{N \times N}$ satisfying (2.1), the derivative of the Struve matrix functions can be obtained by the following matrix differential recurrence relations:

$$
\begin{gathered}
\frac{d}{d z}\left[z^{-A} \mathbf{H}_{A}(z)\right]=\frac{2^{-A}}{\sqrt{\pi}} \Gamma^{-1}\left(A+\frac{3}{2} I\right)-z^{-A} \mathbf{H}_{A+I}(z), \\
\frac{d}{d z}\left[z^{A} \mathbf{H}_{A}(z)\right]=z^{A} \mathbf{H}_{A-I}(z)
\end{gathered}
$$

and

$$
\mathbf{H}_{A-I}(z)-\mathbf{H}_{A+I}(z)=2 D \mathbf{H}_{A}(z)-\frac{1}{\sqrt{\pi}} \Gamma^{-1}\left(A+\frac{3}{2} I\right)\left(\frac{z}{2}\right)^{A} .
$$


Proof. Multiplying both sides of (2.2) by $z^{-A}$ and then differentiating each side with respect to $z$, we get

$$
\begin{aligned}
\frac{d}{d z}\left[z^{-A} \mathbf{H}_{A}(z)\right] & =\frac{d}{d z} \sum_{k=0}^{\infty} \frac{(-1)^{k} z^{(2 k+1) I}}{2^{A+(2 k+1) I} \Gamma\left(k+\frac{3}{2}\right)} \Gamma^{-1}\left(A+\left(k+\frac{3}{2}\right) I\right) \\
& =z^{-A} \sum_{k=0}^{\infty} \frac{(-1)^{k} z^{A+2 k I}}{2^{A+2 k I} \Gamma\left(k+\frac{1}{2}\right)} \Gamma^{-1}\left(A+\left(k+\frac{3}{2}\right) I\right) .
\end{aligned}
$$

Replacing $k$ by $k+1$ in the above equation, we get

$$
\begin{aligned}
\frac{d}{d z}\left[z^{-A} \mathbf{H}_{A}(z)\right]= & z^{-A} \sum_{k=-1}^{\infty} \frac{(-1)^{k+1} z^{A+I+(2 k+1) I}}{2^{A+I+(2 k+1) I} \Gamma\left(k+\frac{3}{2}\right)} \Gamma^{-1}\left(A+\left(k+\frac{5}{2}\right) I\right) \\
= & z^{-A} \frac{\Gamma^{-1}\left(A+\frac{3}{2} I\right) z^{A}}{\Gamma\left(\frac{1}{2}\right)} 2^{-A} \\
& -z^{-A} \sum_{k=0}^{\infty} \frac{(-1)^{k}}{\Gamma\left(k+\frac{3}{2}\right)}\left(\frac{z}{2}\right)^{A+I+(2 k+1) I} \Gamma^{-1}\left(A+\left(k+\frac{5}{2}\right) I\right) \\
= & \frac{\Gamma^{-1}\left(A+\frac{3}{2} I\right)}{\sqrt{\pi}} 2^{-A}-z^{-A} \mathbf{H}_{A+I}(z) .
\end{aligned}
$$

Thus, for the Struve matrix functions, we have the first property

$$
\frac{d}{d z}\left[z^{-A} \mathbf{H}_{A}(z)\right]=\frac{2^{-A}}{\sqrt{\pi}} \Gamma^{-1}\left(A+\frac{3}{2} I\right)-z^{-A} \mathbf{H}_{A+I}(z) .
$$

Moreover, by (2.5), we can write

$$
z \mathbf{H}_{A}^{\prime}(z)=A \mathbf{H}_{A}(z)+\frac{z^{A+I} \Gamma^{-1}\left(A+\frac{3}{2} I\right)}{\sqrt{\pi}} 2^{-A}-z \mathbf{H}_{A+I}(z) .
$$

Multiplying both sides of (2.2) by $z^{A}$ and then differentiating each side with respect to $z$, we get

$$
\begin{aligned}
\frac{d}{d z}\left[z^{A} \mathbf{H}_{A}(z)\right] & =2 \sum_{k=0}^{\infty} \frac{(-1)^{k} \Gamma^{-1}\left(A+\left(k+\frac{3}{2}\right) I\right)\left(A+\left(k+\frac{1}{2}\right) I\right) z^{2 A+2 k I}}{2^{A+(2 k+1) I} \Gamma\left(k+\frac{3}{2}\right)} \\
& =z^{A} \sum_{k=0}^{\infty} \frac{(-1)^{k} \Gamma^{-1}\left(A+\left(k+\frac{1}{2}\right) I\right) z^{A+2 k I}}{2^{A+2 k I} \Gamma\left(k+\frac{3}{2}\right)}=z^{A} \mathbf{H}_{A-I}(z) .
\end{aligned}
$$

Thus, we obtain the second property (2.6). Developing the left-hand side of (2.6) can also be put as

$$
z \mathbf{H}_{A}^{\prime}(z)=z \mathbf{H}_{A-I}(z)-A \mathbf{H}_{A}(z) .
$$

From (2.8) and (2.9), we obtain (2.7).

Theorem 2.3. For matrices $A$ and $A-I$ in $\mathbb{C}^{N \times N}$ satisfying (2.1), the Struve matrix functions satisfy the interesting pure recurrence relation

$$
z \mathbf{H}_{A-I}(z)+z \mathbf{H}_{A+I}(z)=2 A \mathbf{H}_{A}(z)+\frac{z}{\sqrt{\pi}} \Gamma^{-1}\left(A+\frac{3}{2} I\right)\left(\frac{z}{2}\right)^{A} .
$$


Proof. Eliminate $\mathbf{H}_{A}^{\prime}(z)$ from (2.8) and (2.9) to obtain (2.10).

Corollary 2.1. A Struve matrix functions is a solutions of the second-order Struve matrix differential equation

$$
\left(z^{2} D^{2} I+z D I+z^{2} I-A^{2}\right) \mathbf{H}_{A}(z)=\frac{4}{\sqrt{\pi}} \Gamma^{-1}\left(A+\frac{1}{2} I\right)\left(\frac{z}{2}\right)^{A+I} .
$$

Proof. Starting by (2.8) and (2.9), we obtain the following rising and lowering operators:

$$
\widehat{E}_{+} \mathbf{H}_{A}(z)=\mathbf{H}_{A+I}(z)-\frac{1}{\sqrt{\pi}} \Gamma^{-1}\left(A+\frac{3}{2} I\right)\left(\frac{z}{2}\right)^{A},
$$

and

$$
\widehat{E}_{-} \mathbf{H}_{A}(z)=\mathbf{H}_{A-I}(z) \text {. }
$$

From (2.12) and (2.13), Struve matrix functions can be rewritten as

$$
\widehat{E}_{+} \widehat{E}_{-} \mathbf{H}_{A}(z)=\mathbf{H}_{A}(z)-\frac{1}{\sqrt{\pi}} \Gamma^{-1}\left(A+\frac{1}{2} I\right)\left(\frac{z}{2}\right)^{A-I} .
$$

By applying in differential terms the last equation can be rewritten as

$$
\left(\frac{1}{z}(A-I)-D I\right)\left(\frac{1}{z} A+D I\right) \mathbf{H}_{A}(z)=\mathbf{H}_{A}(z)-\frac{1}{\sqrt{\pi}} \Gamma^{-1}\left(A+\frac{1}{2} I\right)\left(\frac{z}{2}\right)^{A-I} .
$$

Thus, we obtain the Struve matrix differential equation (2.11).

Corollary 2.2. The hypergeometric matrix function of a Struve matrix function $\mathbf{H}_{A}(z)$ is of the form

$$
\mathbf{H}_{A}(z)=\frac{2}{\sqrt{\pi}}\left(\frac{z}{2}\right)^{A+I} \Gamma^{-1}\left(A+\frac{3}{2} I\right){ }_{1} F_{2}\left(I ; A+\frac{3}{2} I, \frac{3}{2} I ;-\frac{1}{4} z^{2}\right) .
$$

Proof. It is enough to use (2.2) and (1.2), then we obtain (2.16).

In order to obtain some matrix recurrence relations, we need the following lemma. If the hypergeometric matrix function given by (1.3) is rearranged, we have the next lemma.

Lemma 2.1. For the hypergeometric matrix function ${ }_{1} F_{2}$ we have the equalities

$$
\frac{d^{n}}{d z^{n}}\left[z^{C-I}{ }_{1} F_{2}(A ; B, C ; z)\right]=(C-n I)_{n} z^{C-(n+1) I}{ }_{1} F_{2}(A ; B, C-n I ; z),
$$

where $B, C$ and $C-n I$ are invertible matrices for every integer $n \geq 0$,

$$
\frac{d^{n}}{d z^{n}}\left[z^{B-I}{ }_{1} F_{2}(A ; B, C ; z)\right]=(B-n I)_{n} z^{B-(n+1) I}{ }_{1} F_{2}(A ; B-n I, C ; z),
$$

where $B, C$ and $B-n I$ are invertible matrices for every integer $n \geq 0$, and

$$
\begin{aligned}
\frac{d^{n}}{d z^{n}}\left[{ }_{1} F_{2}(A ; B, C ; z)\right]= & (A)_{n}\left[(B)_{n}\right]^{-1}\left[(C)_{n}\right]^{-1} \\
& \times{ }_{1} F_{2}(A+n I ; B+n I, C+n I ; z),
\end{aligned}
$$

where $C, B, B+n I$ and $C+n I$ are invertible matrices for all integers $n \geq 0$. 
Proof. From (1.2) and (1.8), we get

$$
\frac{d^{n}}{d z^{n}}\left[z^{C+(k-1) I}\right]=(C)_{k}(C-n I)_{n}\left[(C-n I)_{k}\right]^{-1} z^{C+(k-n-1) I} .
$$

Substituting the above relation into the series expression of the hypergeometric matrix function ${ }_{1} F_{2}$, we obtain (2.17).

The proof of Eq. (2.18) is very similar to the proof of Eq. (2.17). Similarly, we get

$$
\begin{aligned}
& \frac{d}{d z}{ }_{1} F_{2}(A ; B, C ; z)=\sum_{k=1}^{\infty} \frac{k z^{k-1}}{k !}(A)_{k}\left[(B)_{k}\right]^{-1}\left[(C)_{k}\right]^{-1} \\
& =\sum_{k=0}^{\infty} \frac{z^{k}}{k !}(A)_{k+1}\left[(B)_{k+1}\right]^{-1}\left[(C)_{k+1}\right]^{-1} \\
& =A B^{-1} C^{-1} \sum_{k=0}^{\infty} \frac{z^{k}}{k !}(A+I)_{k}\left[(B+I)_{k}\right]^{-1}\left[(C+I)_{k}\right]^{-1} \\
& =A B^{-1} C^{-1}{ }_{2} F_{1}(A+I ; B+I, C+I ; z) .
\end{aligned}
$$

By iteration of (2.20), one gets (2.19).

Theorem 2.4. Let $A$ and $A-n I$ be matrices in $\mathbb{C}^{N \times N}$ for $n \in \mathbb{N} \cup\{0\}$ satisfying (2.1) whose eigenvalues $\mu$ all satisfy $\operatorname{Re}(\mu)>-\frac{3}{2}$. The derivative formulas for Struve matrix functions satisfy the following:

$$
\begin{aligned}
\text { (i) }\left(\frac{1}{z} \frac{d}{d z}\right)^{n}\left[z^{A} \mathbf{H}_{A}(z)\right] & =z^{A-n I} \mathbf{H}_{A-n I}(z) \\
\left(\text { ii) } ( \frac { 1 } { z } \frac { d } { d z } ) ^ { n } \left[\left(z^{-A} \mathbf{H}_{A}(z)\right]=\right.\right. & \frac{2^{n I-A}}{\sqrt{\pi}} \Gamma^{-1}\left(A+\frac{3}{2} I\right)\left(\left(\frac{3}{2}-n\right) I\right)_{n} z^{(1-2 n) I} \\
& \times{ }_{1} F_{2}\left(I ;\left(\frac{3}{2}-n\right) I, A+\frac{3}{2} I ;-\frac{z^{2}}{4}\right)
\end{aligned}
$$

where $\left(\frac{3}{2}-n\right) I$ and $A+\frac{3}{2} I$ are invertible matrices in $\mathbb{C}^{N \times N}$.

$$
\begin{aligned}
& \left(\frac{1}{z} \frac{d}{d z}\right)^{n}\left[z^{-A-I} \mathbf{H}_{A}(z)\right]=n ! 2^{A+I} \Gamma^{-1}\left(n+\frac{3}{2}\right) \Gamma^{-1}\left(A+\left(n+\frac{3}{2}\right) I\right) \\
& \times{ }_{1} F_{2}\left((n+1) I ;\left(n+\frac{3}{2}\right) I, A+\left(n+\frac{3}{2}\right) I ;-\frac{1}{4} z^{2}\right),
\end{aligned}
$$

where $A+\left(n+\frac{3}{2}\right) I$ and $\left(n+\frac{3}{2}\right) I$ are invertible matrices in $\mathbb{C}^{N \times N}$.

Proof. (i) To prove (i), take $A \rightarrow I, B \rightarrow \frac{3}{2} I, C \rightarrow A+\frac{3}{2} I$ and $z \rightarrow-\frac{z^{2}}{4}$ in Equation (2.17).

(ii) Take $z \rightarrow-\frac{1}{4} z^{2}, A \rightarrow I, B \rightarrow \frac{3}{2} I$ and $C \rightarrow A+\frac{3}{2} I$ in equation (2.18).

(iii) Take $z \rightarrow-\frac{1}{4} z^{2}, A \rightarrow I, B \rightarrow \frac{3}{2} I$ and $C \rightarrow A+\frac{3}{2} I$ in equation (2.19). 
Now, we derive the integral representations for Struve matrix functions as given in $(2.2)$.

Theorem 2.5. If $A$ is a matrix in $\mathbb{C}^{N \times N}$ satisfying $\operatorname{Re}(\mu)>-\frac{1}{2}$ for all eigenvalues $\mu \in \sigma(A)$, then we have the integral representations for the Struve matrix function

$$
\mathbf{H}_{A}(z)=2\left(\frac{z}{2}\right)^{A} \frac{\Gamma^{-1}\left(A+\frac{1}{2} I\right)}{\sqrt{\pi}} \int_{0}^{1}\left(1-t^{2}\right)^{A-\frac{1}{2} I} \sin (z t) d t .
$$

Proof. If we substitute the series for $\sin (z t)$ on the right-hand side of $(2.24)$, we obtain

$$
\begin{aligned}
& 2\left(\frac{z}{2}\right)^{A} \frac{\Gamma^{-1}\left(A+\frac{1}{2} I\right)}{\sqrt{\pi}} \int_{0}^{1}\left(1-t^{2}\right)^{A-\frac{1}{2} I} \sin (z t) d t \\
& =2\left(\frac{z}{2}\right)^{A} \frac{\Gamma^{-1}\left(A+\frac{1}{2} I\right)}{\sqrt{\pi}} \sum_{k=0}^{\infty} \frac{(-1)^{k} z^{(2 k+1) I}}{(2 k+1) !} \int_{0}^{1}\left(1-t^{2}\right)^{A-\frac{1}{2} I} t^{(2 k+1) I} d t .
\end{aligned}
$$

Taking $u \rightarrow t^{2}$ and using the well-known formula relating the Beta matrix function with the Gamma matrix function, we get

$$
\begin{aligned}
& 2\left(\frac{z}{2}\right)^{A} \frac{\Gamma^{-1}\left(A+\frac{1}{2} I\right)}{\sqrt{\pi}} \sum_{k=0}^{\infty} \frac{(-1)^{k} z^{(2 k+1) I}}{2(2 k+1) !} \int_{0}^{1}(1-u)^{A-\frac{1}{2} I} u^{k I} d u \\
& =\left(\frac{z}{2}\right)^{A} \frac{\Gamma^{-1}\left(A+\frac{1}{2} I\right)}{\sqrt{\pi}} \sum_{k=0}^{\infty} \frac{(-1)^{k} z^{(2 k+1) I}}{(2 k+1) !} \mathbf{B}\left(A+\frac{1}{2} I,(k+1) I\right) \\
& =\sum_{k=0}^{\infty} \frac{(-1)^{k} 2^{2 k+1} k !}{(2 k+1) !} \Gamma^{-1}\left(A+\left(k+\frac{3}{2}\right) I\right)\left(\frac{z}{2}\right)^{A+(2 k+1) I} \\
& =\sum_{k=0}^{\infty} \frac{(-1)^{k}}{\Gamma\left(k+\frac{3}{2}\right)} \Gamma^{-1}\left(A+\left(k+\frac{3}{2}\right) I\right)\left(\frac{z}{2}\right)^{A+(2 k+1) I}=\mathbf{H}_{A}(z) .
\end{aligned}
$$

Theorem 2.6. For matrices $A$ and $A-I$ in $\mathbb{C}^{N \times N}$ satisfying Condition (2.1) whose eigenvalues $\mu$ all satisfy $\operatorname{Re}(\mu)>-\frac{3}{2}$, the integral representations for Struve matrix functions of interest are

$$
\text { (i) } \int_{0}^{z} t^{A} \mathbf{H}_{A-I}(t) d t=z^{A} \mathbf{H}_{A}(z) \text {. }
$$

(ii) $\quad \int_{0}^{z} t^{-A} \mathbf{H}_{A+I}(t) d t=\frac{z}{\sqrt{\pi}} 2^{-A} \Gamma^{-1}\left(A+\frac{3}{2} I\right)-z^{-A} \mathbf{H}_{A}(z)$. 
Proof. Substituting (2.2) in (2.25), we have

$$
\begin{aligned}
& \int_{0}^{z} t^{A} \mathbf{H}_{A-I}(t) d t=\sum_{k=0}^{\infty} \frac{(-1)^{k}}{\Gamma\left(k+\frac{3}{2}\right)} \Gamma^{-1}\left(A+\left(k+\frac{1}{2}\right) I\right)\left(\frac{1}{2}\right)^{A+2 k I} \int_{0}^{z} t^{2 A+2 k I} d t \\
& =\sum_{k=0}^{\infty} \frac{(-1)^{k}}{\Gamma\left(k+\frac{3}{2}\right)} \Gamma^{-1}\left(A+\left(k+\frac{1}{2}\right) I\right)\left(\frac{1}{2}\right)^{A+2 k I}(2 A+(2 k+1) I)^{-1} z^{2 A+(2 k+1) I} \\
& =z^{A} \sum_{k=0}^{\infty} \frac{(-1)^{k}}{\Gamma\left(k+\frac{3}{2}\right)} \Gamma^{-1}\left(A+\left(k+\frac{3}{2}\right) I\right)\left(\frac{z}{2}\right)^{A+(2 k+1) I}=z^{A} \mathbf{H}_{A}(z) .
\end{aligned}
$$

Similarly, the proof of the equation (2.26) is very similar to the proof of equation (2.25), thus we get the desired results.

Theorem 2.7. If $A$ and $B$ are matrices in $\mathbb{C}^{N \times N}$, the integral representation for Struve matrix functions in (2.2) holds true:

$$
\begin{aligned}
& \int_{0}^{z} t^{B} \mathbf{H}_{A}(t) d t=\frac{1}{\sqrt{\pi}} 2^{-A} z^{A+B+2 I}(A+B+2 I)^{-1} \Gamma^{-1}\left(A+\frac{3}{2} I\right) \\
& \quad \times{ }_{2} F_{3}\left(I, \frac{1}{2}(B+A)+I ; \frac{3}{2} I, A+\frac{3}{2} I, \frac{1}{2}(B+A)+2 I ;-\frac{1}{4} z^{2}\right)
\end{aligned}
$$

where $A$ is a matrix in $\mathbb{C}^{N \times N}$ satisfying Conditions (2.1), and $\frac{1}{2}(B+A)+2 I$ is an invertible matrix in $\mathbb{C}^{N \times N}$ satisfying the condition $\operatorname{Re}(\mu)>-2$ for all eigenvalues $\mu \in \sigma(A+B)$.

Proof. From (2.2), the left-hand side of (2.27) can be written as

$$
\begin{aligned}
\int_{0}^{\infty} t^{B} \mathbf{H}_{A}(t) d t= & \sum_{k=0}^{\infty} \frac{(-1)^{k}}{\Gamma\left(k+\frac{3}{2}\right)} \Gamma^{-1}\left(A+\left(k+\frac{3}{2}\right) I\right) \\
& \times\left(\frac{1}{2}\right)^{A+(2 k+1) I} \int_{0}^{z} t^{A+B+(2 k+1) I} d t .
\end{aligned}
$$

Taking

$$
\begin{aligned}
(A+B+2(k+1) I)^{-1}= & \frac{1}{2}\left(\frac{1}{2}(A+B)+I\right)^{-1}\left[\left(\frac{1}{2}(A+B)+2 I\right)_{k}\right]^{-1} \\
& \times\left(\frac{1}{2}(A+B)+I\right)_{k}
\end{aligned}
$$

and using the hypergeometric matrix function with the help of matrix functional calculus, we have 


$$
\begin{aligned}
& \sum_{k=0}^{\infty} \frac{(-1)^{k}}{\Gamma\left(k+\frac{3}{2}\right)} \Gamma^{-1}\left(A+\left(k+\frac{3}{2}\right) I\right)\left(\frac{1}{2}\right)^{A+(2 k+1) I}(A+B+2(k+1) I)^{-1} \\
& \times z^{A+B+(2 k+2) I}=\Gamma^{-1}\left(\frac{3}{2}\right) 2^{-A-I} z^{A+B+2 I} \Gamma^{-1}\left(A+\frac{3}{2} I\right) \\
& \times \sum_{k=0}^{\infty} \frac{(-1)^{k}(I)_{k}}{k !}\left[\left(\frac{3}{2} I\right)_{k}\right]^{-1}\left[\left(A+\frac{3}{2} I\right)_{k}\right]^{-1}\left[\left(\frac{1}{2}(A+B)+2 I\right)_{k}\right]^{-1}\left(\frac{1}{2}(A+B)+I\right)_{k}\left(\frac{z}{2}\right)^{2 k I} \\
& \times \frac{1}{2}\left(\frac{1}{2}(A+B)+I\right)^{-1}\left[\left(A+\frac{3}{2} I\right)\right. \\
& =\frac{1}{\sqrt{\pi}} 2^{-A} z^{A+B+2 I}(A+B+2 I)^{-1} \Gamma^{-1}(A) \\
& \times{ }_{2} F_{3}\left(I, \frac{1}{2}(B+A)+I ; \frac{3}{2} I, A+\frac{3}{2} I, \frac{1}{2}(B+A)+2 I ;-\frac{1}{4} z^{2}\right) .
\end{aligned}
$$

Theorem 2.8. If $A$ and $B$ are matrices in $\mathbb{C}^{N \times N}$ satisfying (2.1) and $A-B$ is a positive stable matrix, we have the formula

$$
\mathbf{H}_{A}(z)=2 \Gamma^{-1}(A-B)\left(\frac{z}{2}\right)^{A-B} \int_{0}^{1}\left(1-t^{2}\right)^{A-B-I} t^{B+I} \mathbf{H}_{B}(z t) d t .
$$

Proof. Let us consider the integral

$$
\mathbb{I}=\int_{0}^{1}\left(1-t^{2}\right)^{A-B-I} t^{B+I} \mathbf{H}_{B}(z t) d t,
$$

and substitute the series (2.2) for Struve's matrix function to obtain

$$
\begin{aligned}
\mathbb{I}= & \sum_{k=0}^{\infty} \frac{(-1)^{k}}{\Gamma\left(k+\frac{3}{2}\right)} \Gamma^{-1}\left(B+\left(k+\frac{3}{2}\right) I\right)\left(\frac{z}{2}\right)^{B+(2 k+1) I} \\
& \times \int_{0}^{1}\left(1-t^{2}\right)^{A-B-I} t^{2 B+(2 k+2) I} d t .
\end{aligned}
$$

Substituting $u \rightarrow t^{2}$ and using the well known formula relating the Beta matrix function with the Gamma matrix function, we get

$$
\begin{aligned}
\mathbb{I} & =\sum_{k=0}^{\infty} \frac{(-1)^{k}}{2 \Gamma\left(k+\frac{3}{2}\right)} \Gamma^{-1}\left(B+\left(k+\frac{3}{2}\right) I\right)\left(\frac{z}{2}\right)^{B+(2 k+1) I} \int_{0}^{1}(1-u)^{A-B-I} u^{B+\left(k+\frac{1}{2}\right) I} d u \\
& =\sum_{k=0}^{\infty} \frac{(-1)^{k}}{2 \Gamma\left(k+\frac{3}{2}\right)} \Gamma^{-1}\left(B+\left(k+\frac{3}{2}\right) I\right)\left(\frac{z}{2}\right)^{B+(2 k+1) I} \mathbf{B}\left(A-B, B+\left(k+\frac{3}{2}\right) I\right) \\
& =\frac{1}{2} \Gamma(A-B)\left(\frac{z}{2}\right)^{B-A} \sum_{k=0}^{\infty} \frac{(-1)^{k}}{\Gamma\left(k+\frac{3}{2}\right)}\left(\frac{z}{2}\right)^{A+(2 k+1) I} \Gamma^{-1}\left(A+\left(k+\frac{3}{2}\right) I\right) \\
& =\frac{1}{2} \Gamma(A-B)\left(\frac{z}{2}\right)^{B-A} \mathbf{H}_{A}(z) .
\end{aligned}
$$




\section{Modified Struve matrix functions: Definition and some new relations}

Definition 3.1. Let $A$ be a matrix in $\mathbb{C}^{N \times N}$ satisfying (2.1). We define the modified Struve matrix function

$$
\mathbf{L}_{A}(z)=\sum_{k=0}^{\infty} \frac{1}{\Gamma\left(k+\frac{3}{2}\right)} \Gamma^{-1}\left(A+\left(k+\frac{3}{2}\right) I\right)\left(\frac{z}{2}\right)^{A+(2 k+1) I} .
$$

In a similar manner as in the proof of Theorems 2.2 and 2.6 one gets the following results for the modified Struve matrix function $\mathbf{L}_{A}(z)$ by taking the matrix $A$ satisfying Condition (2.1) which bears the same relations to Struve's matrix function, respectively.

Theorem 3.1. For matrices $A, A-I$ and $A-m I$ in $\mathbb{C}^{N \times N}$ satisfying (2.1) and for $m \in \mathbb{N}$, the modified Struve matrix functions satisfy the matrix recurrence relations

$$
\begin{gathered}
\mathbf{L}_{A-I}(z)+\mathbf{L}_{A+I}(z)=2 D \mathbf{L}_{A}(z)-\frac{1}{\sqrt{\pi}} \Gamma^{-1}\left(A+\frac{3}{2} I\right)\left(\frac{z}{2}\right)^{A}, \\
\mathbf{L}_{A-I}(z)-\frac{1}{z} A \mathbf{L}_{A}(z)=D \mathbf{L}_{A}(z), \\
\frac{d}{d z}\left[z^{-A} \mathbf{L}_{A}(z)\right]=\frac{2^{-A}}{\sqrt{\pi}} \Gamma^{-1}\left(A+\frac{3}{2} I\right)+z^{-A} \mathbf{L}_{A+I}(z), \\
\frac{d}{d z}\left[z^{A} \mathbf{L}_{A}(z)\right]=z^{A} \mathbf{L}_{A-I}(z)
\end{gathered}
$$

and

$$
\left(\frac{1}{z} \frac{d}{d z}\right)^{m}\left(z^{A} \mathbf{L}_{A}(z)\right)=z^{A-m I} \mathbf{L}_{A-m I}(z) .
$$

Now by (3.1), we note without proof the following result for modified Struve matrix functions via Theorem 2.3:

Theorem 3.2. For matrices $A$ and $A-I$ in $\mathbb{C}^{N \times N}$ satisfying (2.1), the modified Struve matrix functions satisfy the interesting relation

$$
\mathbf{L}_{A-I}(z)-\mathbf{L}_{A+I}(z)=\frac{2}{z} A \mathbf{L}_{A}(z)+\frac{1}{\sqrt{\pi}} \Gamma^{-1}\left(A+\frac{3}{2} I\right)\left(\frac{z}{2}\right)^{A} .
$$

In a similar manner as in the proof of Theorem 2.8 , one can easily get the next results.

Theorem 3.3. If $A$ and $A-I$ are matrices in $\mathbb{C}^{N \times N}$ satisfying (2.1), the integrals for the modified Struve matrix function of interest are

$$
\begin{gathered}
\text { (i) } \int_{0}^{z} t^{A} \mathbf{L}_{A-I}(t) d t=z^{A} \mathbf{L}_{A}(z) . \\
\text { (ii) } \int_{0}^{z} t^{-A} \mathbf{L}_{A+I}(t) d t=z^{-A} \mathbf{L}_{A}(z)-\frac{z}{\sqrt{\pi}} 2^{-A} \Gamma^{-1}\left(A+\frac{3}{2} I\right) .
\end{gathered}
$$


Theorem 3.4. If $A$ is a matrix in $\mathbb{C}^{N \times N}$ satisfying (2.1), the following integral representations of interest are valid:

$$
\text { (i) } \int_{0}^{\infty} e^{-t} t^{A} \mathbf{L}_{A}(t) d t=-\frac{2^{A+I}}{\pi}(2 A+1)^{-1} \Gamma(A+I),
$$

where $-1<\operatorname{Re}(\mu)<-\frac{1}{2}$ for all eigenvalues $\mu \in \sigma(A)$.

$$
\text { (ii) } \int_{0}^{\infty} e^{-t} t^{-A} \mathbf{L}_{A}(t) d t=\frac{2^{I-A}}{\sqrt{\pi}}(2 A-1)^{-1} \Gamma^{-1}\left(A+\frac{1}{2} I\right) \text {, }
$$

where $\operatorname{Re}(\mu)>\frac{1}{2}$ for all eigenvalues $\mu \in \sigma(A)$.

Proof. From (3.1), the left-hand side of (3.11), and using Gamma matrix function with the help of the equations in (1.4), (1.9) and (1.10), we get

$$
\begin{aligned}
& \int_{0}^{\infty} e^{-t} t^{A} \mathbf{L}_{A}(t) d t \\
& =\sum_{k=0}^{\infty} \frac{1}{\Gamma\left(k+\frac{3}{2}\right)} \Gamma^{-1}\left(A+\left(k+\frac{3}{2}\right) I\right)\left(\frac{1}{2}\right)^{A+(2 k+1) I} \int_{0}^{\infty} e^{-t} t^{2 A+(2 k+1) I} d t \\
& =\sum_{k=0}^{\infty} \frac{1}{\Gamma\left(k+\frac{3}{2}\right)} \Gamma^{-1}\left(A+\left(k+\frac{3}{2}\right) I\right)\left(\frac{1}{2}\right)^{A+(2 k+1) I} \Gamma(2 A+(2 k+2) I) \\
& =\frac{2^{A}}{\sqrt{\pi}} \sum_{k=0}^{\infty} \frac{1}{\Gamma\left(k+\frac{3}{2}\right)} \Gamma(A+(k+1) I) \\
& =\frac{2^{A}}{\sqrt{\pi} \Gamma\left(\frac{3}{2}\right)} \Gamma(A+I) \sum_{k=0}^{\infty}(A+I)\left[\left(\frac{3}{2} I\right)_{k}\right]_{k}^{-1} \\
& =\frac{2^{A+I}}{\pi} \Gamma(A+I){ }_{2} F_{1}\left(I, A+I ; \frac{3}{2} I ; 1\right)=-\frac{2^{A+I}}{\pi} \Gamma(A+I)(2 A+I)^{-1} .
\end{aligned}
$$

From (3.1), the left-hand side of (3.11) and using Gamma matrix function and Theorem 1.1, we get

$$
\begin{aligned}
& \int_{0}^{\infty} e^{-t} t^{-A} \mathbf{L}_{A}(t) d t \\
& =\sum_{k=0}^{\infty} \frac{1}{\Gamma\left(k+\frac{3}{2}\right)} \Gamma^{-1}\left(A+\left(k+\frac{3}{2}\right) I\right)\left(\frac{1}{2}\right)^{A+(2 k+1) I} \int_{0}^{\infty} e^{-t} t^{(2 k+1) I} d t \\
& =\frac{2^{-A}}{\sqrt{\pi}} \sum_{k=0}^{\infty} \Gamma((k+1) I) \Gamma^{-1}\left(A+\left(k+\frac{3}{2}\right) I\right) \\
& =\frac{2^{-A}}{\sqrt{\pi}} \Gamma^{-1}\left(A+\frac{3}{2} I\right) \sum_{k=0}^{\infty}(I)_{k}\left[\left(A+\frac{3}{2} I\right)_{k}\right]^{-1} \\
& =\frac{2^{-A}}{\sqrt{\pi}} \Gamma^{-1}\left(A+\frac{3}{2} I\right){ }_{2} F_{1}\left(I, I ; A+\frac{3}{2} I ; 1\right)
\end{aligned}
$$




$$
=\frac{2^{I-A}}{\sqrt{\pi}}(2 A-I)^{-1} \Gamma^{-1}\left(A+\frac{1}{2} I\right) .
$$

In a similar manner, using (3.1), (1.4), (1.9) and (1.10), one can get the next result.

Theorem 3.5. The infinite integral representation for modified Struve matrix functions in (3.1) holds:

$$
\int_{0}^{\infty} e^{-t} t^{-A-I} \mathbf{L}_{A}(t) d t=\frac{2^{-A}}{\sqrt{\pi}} A^{-1} \Gamma^{-1}\left(A+\frac{1}{2} I\right) .
$$

\section{New results for Struve and modified Struve matrix functions}

In this section, several new identities and the implicit summation formulae for Struve and modified Struve matrix functions are constructed by using operational techniques for a method and new results for members of the families of these matrix functions.

Theorem 4.1. For matrices $A$ and $A-n I$ in $\mathbb{C}^{N \times N}$ satisfying (2.1) for $n \in \mathbb{N} \cup\{0\}$, the summation formula for Struve matrix functions holds:

$$
(z+2 a)^{\frac{1}{2} A} \mathbf{H}_{A}(\sqrt{z+2 a})=\sum_{n=0}^{\infty} \frac{a^{n}}{n !} z^{\frac{1}{2}(A-n I)} \mathbf{H}_{A-n I}(\sqrt{z}) .
$$

Proof. The action of the shift operator on a function of $x$ produces a shift of the variable by $\alpha$ and thus it considered as follows:

$$
\exp \left(\alpha \frac{d}{d x}\right) f(x)=f(x+\alpha) \text {. }
$$

We consider the operator $\mathbb{A}[16]$ in the following form:

$$
\mathbb{A}=2 \frac{d}{d z} .
$$

Operating on the Struve matrix functions $\mathbf{H}_{A}(\sqrt{z})$ and using the shift operator, we have the formula

$$
e^{a \mathbb{A}}\left[z^{\frac{1}{2} A} \mathbf{H}_{A}(\sqrt{z})\right]=e^{2 a \frac{\partial}{\partial z}}\left[z^{\frac{1}{2} A} \mathbf{H}_{A}(\sqrt{z})\right]=(z+2 a)^{\frac{1}{2} A} \mathbf{H}_{A}(\sqrt{z+2 a}) .
$$

On the other hand, we get

$$
e^{a \mathbb{A}}\left[z^{\frac{1}{2} A} \mathbf{H}_{A}(\sqrt{z})\right]=\sum_{k=0}^{\infty} \frac{a^{k}}{k !} \mathbb{A}^{k}\left[z^{\frac{1}{2} A} \mathbf{H}_{A}(\sqrt{z})\right] .
$$

Combining these equations (4.3) and (4.4), we get

$$
(z+2 a)^{\frac{1}{2} A} \mathbf{H}_{A}(\sqrt{z+2 a})=\sum_{k=0}^{\infty} \frac{a^{k}}{k !} \mathbb{A}^{k}\left[z^{\frac{1}{2} A} \mathbf{H}_{A}(\sqrt{z})\right] .
$$


From (2.21) and taking $z \rightarrow \sqrt{z}$, we rewrite the expansion formula in the form

$$
\frac{d^{n}}{d z^{n}}\left[z^{\frac{1}{2} A} \mathbf{H}_{A}(\sqrt{z})\right]=\frac{1}{2^{n}} z^{\frac{1}{2}(A-n I)} \mathbf{H}_{A-n I}(\sqrt{z}),
$$

and

$$
\mathbb{A}^{n}\left[z^{\frac{1}{2} A} \mathbf{H}_{A}(\sqrt{z})\right]=z^{\frac{1}{2}(A-n I)} \mathbf{H}_{A-n I}(\sqrt{z}) .
$$

Performing the operation (4.4) and using operational definition (4.7), we find

$$
\sum_{n=0}^{\infty} \frac{a^{n}}{n !} \mathbb{A}^{n}\left[z^{\frac{1}{2} A} \mathbf{H}_{A}(\sqrt{z})\right]=\sum_{n=0}^{\infty} \frac{a^{n}}{n !} z^{\frac{1}{2}(A-n I)} \mathbf{H}_{A-n I}(\sqrt{z}) .
$$

From (4.5) and (4.8), we obtain (4.1).

Theorem 4.2. The Struve and modified Struve matrix functions satisfy the following differential relations:

$$
\frac{d^{n}}{d z^{n}}\left[z^{\frac{1}{2} A} \mathbf{L}_{A}(\sqrt{z})\right]=\frac{1}{2^{n}} z^{\frac{1}{2}(A-n I)} \mathbf{L}_{A-n I}(\sqrt{z}),
$$

where $A$ and $A-n I$ are matrices in $\mathbb{C}^{N \times N}$ satisfying (2.1) for $n \in \mathbb{N} \cup\{0\}$,

$$
\begin{aligned}
\frac{d^{n}}{d z^{n}}\left[z^{-\frac{1}{2} A} \mathbf{H}_{A}(\sqrt{z})\right]= & \frac{2^{n I-A}}{\sqrt{\pi}} \Gamma^{-1}\left(A+\frac{3}{2} I\right)\left(\left(\frac{3}{2}-n\right) I\right)_{n} z^{\frac{1-2 n}{2} I} \\
& \times{ }_{1} F_{2}\left(I ;\left(\frac{3}{2}-n\right) I, A+\frac{3}{2} I ;-\frac{z}{4}\right),
\end{aligned}
$$

where $A+\frac{3}{2} I$ and $\left(\frac{3}{2}-n\right) I$ are invertible matrices in $\mathbb{C}^{N \times N}$, and

$$
\begin{aligned}
& \frac{d^{n}}{d z^{n}}\left[z^{-\frac{1}{2}(A+I)} \mathbf{H}_{A}(\sqrt{z})\right]=n ! 2^{A+I} \Gamma^{-1}\left(n+\frac{3}{2}\right) \Gamma^{-1}\left(A+\left(n+\frac{3}{2}\right) I\right) \\
& \times{ }_{1} F_{2}\left((n+1) I ;\left(n+\frac{3}{2}\right) I, A+\left(n+\frac{3}{2}\right) I ;-\frac{1}{4} z\right),
\end{aligned}
$$

where $A+\left(n+\frac{3}{2}\right) I$ and $\left(n+\frac{3}{2}\right) I$ are invertible matrices in $\mathbb{C}^{N \times N}$.

Proof. Again, replacing $z$ by $\sqrt{z}$ in Eqs. (2.22), (2.23) and (3.6), and taking into account that the matrices $A$ and $A-n I$ satisfy Condition (2.1) for $n \in \mathbb{N} \cup\{0\}$, we get the relations $(4.9),(4.10)$ and $(4.11)$.

Theorem 4.3. The summation formulae for Struve and modified Struve matrix functions hold:

$$
(z+2 a)^{\frac{1}{2} A} \mathbf{L}_{A}(\sqrt{z+2 a})=\sum_{n=0}^{\infty} \frac{a^{n}}{n !} z^{\frac{1}{2}(A-n I)} \mathbf{L}_{A-n I}(\sqrt{z})
$$


where $A-n I$ and $A$ are matrices in $\mathbb{C}^{N \times N}$ satisfying (2.1) for $n \in \mathbb{N} \cup\{0\}$,

$$
\begin{aligned}
& (z+2 a)^{-\frac{1}{2} A} \mathbf{H}_{A}(\sqrt{z+2 a})=\sum_{n=0}^{\infty} \frac{a^{n}}{n !} \frac{2^{n I-A}}{\sqrt{\pi}} \Gamma^{-1}\left(A+\frac{3}{2} I\right) \\
& \times\left(\left(\frac{3}{2}-n\right) I\right)_{n} z^{\frac{1-2 n}{2} I}{ }_{1} F_{2}\left(I ;\left(\frac{3}{2}-n\right) I, A+\frac{3}{2} I ;-\frac{z}{4}\right),
\end{aligned}
$$

where $\left(\frac{3}{2}-n\right) I$ and $A+\frac{3}{2} I$ are invertible matrices in $\mathbb{C}^{N \times N}$, and

$$
\begin{aligned}
& (z+2 a)^{-\frac{1}{2}(A+I)} \mathbf{H}_{A}(\sqrt{z+2 a})=\sum_{n=0}^{\infty} \frac{a^{n}}{n !} n ! 2^{A+I} \Gamma^{-1}\left(n+\frac{3}{2}\right) \\
& \times \Gamma^{-1}\left(A+\left(n+\frac{3}{2}\right) I\right){ }_{1} F_{2}\left((n+1) I ;\left(n+\frac{3}{2}\right) I, A+\left(n+\frac{3}{2}\right) I ;-\frac{1}{4} z\right),
\end{aligned}
$$

where $A+\left(n+\frac{3}{2}\right) I$ and $\left(n+\frac{3}{2}\right) I$ are invertible matrices in $\mathbb{C}^{N \times N}$.

Proof. In a similar manner by the same lines as in the proof of Theorem 4.1, using the analogous procedure as above by operator $\mathbb{A}(4.2)$ for the Struve and modified Struve matrix functions, we obtain the results (4.12), (4.13) and (4.14).

\section{Concluding Remarks and observations}

The results thus derived in this study are generally extended, and generalized in character. Further research will aim at finding certain applications in the theory of special matrix functions for other new families of Struve and modified Struve matrix functions.

\section{Acknowledgements}

The author thanks the anonymous referees for their useful technical comments and valuable suggestions to improve the readability of the paper, which led to a significant improvement of the paper.

\section{References}

[1] B. Çekim and A. Altin, Matrix analogues of some properties for Bessel matrix functions, Journal of Mathematical Sciences, the University of Tokyo 22 no. 2 (2015), 519-530.

[2] B. Çekim and E. Erkuş-Duman, Integral representations for Bessel matrix functions, Gazi University Journal of Science 27 no. 1 (2014), 663-667.

[3] E. Defez and L. Jódar, Chebyshev matrix polynomails and second order matrix differential equations, Utilitas Mathematica 61 (2002) 107-123.

[4] N. Dunford and J.T. Schwartz, Linear Operators, part I. General Theory, Interscience Publishers, New York, 1957.

[5] L. Jódar and J.C. Cortés, Some properties of gamma and beta matrix functions, Applied Mathematics Letters 11 (1998), 89-93. 
[6] L. Jódar and J.C. Cortés, On the hypergeometric matrix function, Journal of Computational and Applied Mathematics 99 (1998), 205-217.

[7] J. Sastre and L. Jódar, Asymptotics of the modified Bessel and incomplete Gamma matrix functions, Applied Mathematics Letters 16 no. 6 (2003), 815-820.

[8] A. Shehata, A new extension of Bessel matrix functions, Southeast Asian Bulletin of Mathematics 40 no. 2 (2016), 265-288.

[9] A. Shehata, Some relations on the generalized Bessel matrix polynomials, Asian Journal of Mathematics and Computer Research 17 no. 1 (2017), 1-25.

[10] A. Shehata, A note on two-variable Lommel matrix functions, Asian-European Journal of Mathematics (AEJM) 11 no. 1 (2018), 1850041 (14 pp.).

[11] A. Shehata, Some properties associated with the Bessel matrix functions, Konuralp Journal of Mathematics (KJM) 5 no. 2 (2017), 24-35.

[12] A. Shehata, Some relations on generalized Rice's matrix polynomials. An International Journal of Applications and Applied Mathematics 12 no. 1 (2017), 367-391.

[13] A. Shehata, Extended biorthogonal matrix polynomials, Mathematica Moravica 21 no. 2 (2017), 27-50.

[14] A. Shehata and S. Khan, On Bessel-Maitland matrix function, Journal Mathematica (Cluj) 57 (80) no. 1-2 (2015), 90-103.

[15] A.A. Mahmoud, Some Topics in Complex Analysis and its Applications in Special Functions, M.Sc. Thesis, Al-Azhar Assiut University, Assiut, Egypt, 2009.

[16] B. Kaufman, Special functions of mathematical physics from the view point of LieAlgebra, Journal of Mathematics 7 (1966), 447-457.

Ayman Shehata

Department of Mathematics, Faculty of Science, Assiut University, Assiut 71516, Egypts

and

Department of Mathematics, College of Science and Arts in Unaizah, Qassim University, Qassim, Saudi Arabia

e-mail: drshehata2006@yahoo.com

Received: February 9, 2018.

Revised: April 14, 2018.

Accepted: April 24, 2018.

Open Access This article is distributed under the terms of the Creative Commons Attribution 4.0 International License (http://creativecommons.org/licenses/by/4.0/), which permits unrestricted use, distribution, and reproduction in any medium, provided you give appropriate credit to the original author(s) and the source, provide a link to the Creative Commons license, and indicate if changes were made. 